\title{
Shock-Induced Borehole Waves and Fracture Effects
}

\author{
Huajun Fan · D. M. J. Smeulders
}

Received: 4 February 2011 / Accepted: 25 August 2011 / Published online: 6 March 2012

(C) The Author(s) 2012. This article is published with open access at Springerlink.com

\begin{abstract}
We perform wave experiments using a vertical shock tube setup. Shock waves are generated by the rupture of a thin membrane. In the test section, the incident pressure waves generate borehole-guided waves along water-saturated samples. The tube is equipped with side wall gages and a mobile pressure probe, so that the attenuation and reflection of the wave can be measured. The computation for a single horizontal fracture intersecting a vertical borehole gives a quantitative prediction of reflection and transmission of borehole-guided waves. Three different fracture apertures are used for the calculation. Fracture aperture significantly affects both reflection and transmission coefficients. Large fractures increase reflectivity and decrease transmissivity. In the experiment, we found that both pressures above and below the fracture are influenced by the fracture aperture indeed, thus indicating the potential for fracture detection by borehole waves.
\end{abstract}

Keywords Borehole waves · Reflection coefficient · Transmission coefficient . Fracture detection $\cdot$ Shock tube

\section{Introduction}

Fractured reservoir is ubiquitous in hydrocarbon reservoir engineering (Chilingarian et al. 1992; Naimi-Tajdar et al. 2007; Ramirez et al. 2009). The Stoneley wave (St) has been used for the detection and characterization of fracture zones (Hornby et al. 1989; Kostek et al. 1998; Qobi et al. 2001; Saito et al. 2004). The borehole fluid flow into the fracture and scattering at the fracture can lead to attenuation of the St (Hornby et al. 1989; Tang and Cheng 1989). The

This paper has also been published in Volume 91/1, pp. 295-302, doi:10.1007/s11242-011-9845-3 (open access).

H. Fan $(\varangle)$. D. M. J. Smeulders

Department of Geotechnology, Delft University of Technology, Delft, The Netherlands e-mail: h.fan@tudelft.nl

D. M. J. Smeulders

Department of Mechanical Engineering, Eindhoven University of Technology,

Eindhoven, The Netherlands 


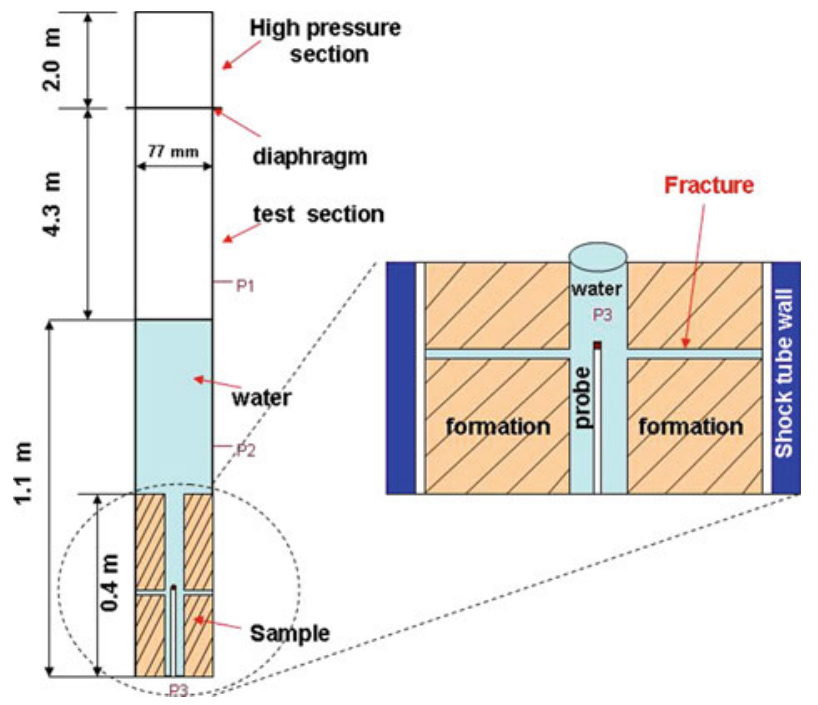

Fig. 1 Schematic of the shock tube setup and fractured sample

relationship between St propagation and permeability was studied by Rosenbaum (1974). Theoretical models of borehole St propagation in permeable materials were evaluated by laboratory experiments by Winkler et al. (1989).

In this article, we will introduce a new experimental technique for fracture detection by means of borehole wave propagation. We use a vertical shock tube to generate a borehole wave in a core sample in the test section of the tube. The shock tube is shown in Fig. 1. The high-pressure and the low-pressure parts are separated by a membrane. The dimensions of each section are indicated in the figure. A cylinder which has a centralized borehole is mounted in the test section of the shock tube. A probe inside the borehole can record the pressures at different positions. Similar experiments were performed by Winkler et al. (1989), but they used a conventional piezoelectric source. The advantage of using shockinduced guided waves is that we cover a wide band of frequencies in one experimental run, and that shock amplitude is easily varied over a wide range of amplitude levels. The frequency band width was investigated in a previous article (Chao et al. 2004) and found to be between 0.5 and $80 \mathrm{kHz}$, approximately. Van der Grinten et al. (1985, 1987), Sniekers et al. (1989), and Smeulders and Van Dongen (1997) also performed shock-tube experiments on water-saturated and partially saturated samples, but here we focus on borehole wave along water-saturated samples.

The tube is filled with water. The membrane is ruptured by an electrical current, and a stepwise shock wave in air is generated. The shock wave then travels downward, partially reflecting, and partially transmitting into the water layer on top of the sample. The transmitted wave reaches the sample where it partially propagates as a borehole wave. The pressures inside the borehole are recorded by the mobile probe P3. The transducers P1 and P2 are mounted in the shock-tube wall. P2 is used to trigger the data recording system (see Fig. 1).

\section{Theoretical Formulation}

We consider a single horizontal fracture, having a width $L$, intersecting the borehole (see Fig. 2). The fracture is of infinite extent in the radial ( $r$ ) direction. The borehole has radius 
Fig. 2 The cylindrical coordinates of the borehole fracture

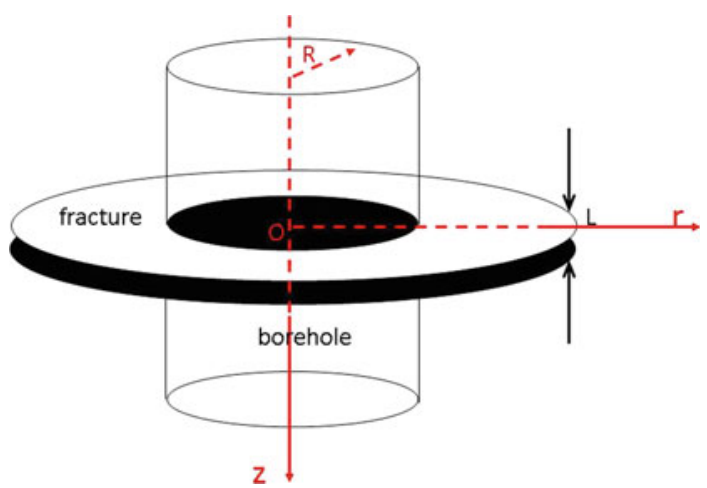

$R$. The plane $z=0$ is in the middle of the fracture. The $z$-coordinate is pointing downward. The maximum frequency attained in our experiments is some $80 \mathrm{kHz}$, which means that the wavelength $\lambda$ is always larger than $2.5 \mathrm{~cm}$, so that for all practical purposes, $\lambda / d \gg 1$. We thus assume that the borehole fluid pressure is uniform across the borehole (Tang and Cheng 1993). The borehole wave equation is

$$
\frac{d^{2} \psi}{d z^{2}}+\kappa^{2} \psi=0,
$$

where $\psi$ is the displacement potential, and $\kappa$ is the wavenumber. The fluid pressure $P$ and the axial fluid displacement $U$ in the borehole are given by

$$
P=\rho_{f} \omega^{2} \psi, U=\frac{d \psi}{d z},
$$

where $\rho_{f}$ is the fluid density, and $\omega$ is the angular frequency. Borehole wave propagation is described by

$$
\begin{aligned}
& \psi=A^{+} e^{i \kappa_{1} z}+A^{-} e^{-i \kappa_{1} z} \text { for } z<-L / 2, \\
& \psi=B^{+} e^{i \kappa_{2} z}+B^{-} e^{-i \kappa_{2} z} \text { for }-L / 2<z<L / 2, \\
& \psi=C^{+} e^{i \kappa_{1} z} \text { for } z>L / 2,
\end{aligned}
$$

In the region $z<-L / 2, A^{+} e^{i \kappa_{1} z}$ and $A^{-} e^{-i \kappa_{1} z}$ represent the incident wave propagating in the positive $z$ direction and the reflected wave propagating in the negative $z$ direction, respectively. Note that $\kappa_{1}$ is the fluid wavenumber in the undisturbed borehole, and $\kappa_{2}$ is the fluid wavenumber where the borehole is intersected by the fracture. $A^{+}$is the incident amplitude, and $A^{-}$is the reflected amplitude. In the region $-L / 2<z<L / 2, B^{+}$and $B^{-}$are the amplitude of the waves propagating in the positive and negative $z$ directions, respectively. $C^{+}$ is the amplitude of the transmitted wave in the region $z>L / 2$. The fluid displacement and the pressure should be continuous at $z=L / 2$ and $z=-L / 2$. The coefficients $A^{-}, B^{+}, B^{-}$ and $C^{+}$can now be calculated as a function of the incident amplitude coefficient term $A^{+}$:

$$
\begin{aligned}
& A^{-} / A^{+}=2 i\left(\kappa_{2}^{2}-\kappa_{1}^{2}\right) \sin \left(\kappa_{2} L\right) / G, \\
& B^{+} / A^{+}=2 \kappa_{1}\left(\kappa_{1}+\kappa_{2}\right) e^{-i \kappa_{2} L} / G, \\
& B^{-} / A^{+}=2 \kappa_{1}\left(\kappa_{2}-\kappa_{1}\right) e^{i \kappa_{2} L} / G, \\
& C^{+} / A^{+}=4 \kappa_{1} \kappa_{2} e^{-i \kappa_{1} L} / G,
\end{aligned}
$$




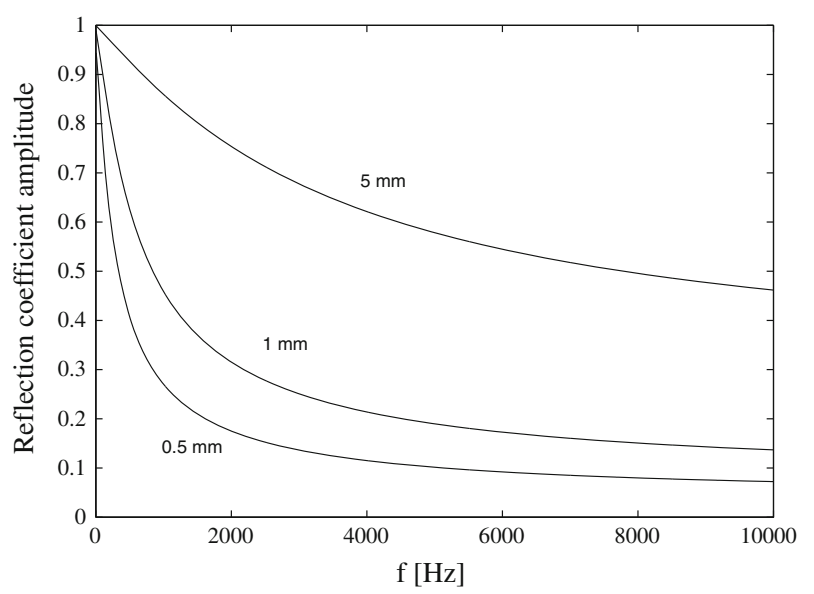

Fig. 3 Amplitude of reflection coefficient above the fracture at three different fracture apertures

where $G$ is given by

$$
G=\left(\kappa_{1}+\kappa_{2}\right)^{2} e^{-i \kappa_{2} L}-\left(\kappa_{1}-\kappa_{2}\right)^{2} e^{i \kappa_{2} L} .
$$

The above equations were also found by Tang and Cheng (1993).

Assuming a rigid formation, the wavenumber in the region $z<-L / 2$ and $z>L / 2$ is simply $\kappa_{1}=\omega / C_{f}=\omega \sqrt{\rho_{f} / K_{f}}$, where $C_{f}$ is acoustic velocity in the fluid, and $K_{f}$ is the fluid bulk modulus. The wavenumber $\kappa$ in the fracture is given by the fracture dispersion equation (Tang and Cheng 1989):

$$
\kappa^{2} \tan \left(\frac{L}{2} \bar{f}\right)+f \bar{f} \tan \left(\frac{L}{2} f\right)=0 .
$$

The parameters $f$ and $\bar{f}$ can be expressed as follows:

$$
\begin{aligned}
f^{2} & =\frac{\omega^{2}}{C_{f}^{2}-\frac{4}{3} i \omega \nu}-\kappa^{2}, \\
\bar{f}^{2} & =\frac{\omega}{-i \nu}-\kappa^{2} .
\end{aligned}
$$

The kinematic viscosity $v=\mu / \rho_{f}$, with $\mu$ the dynamic viscosity. The effective wavenumber $\kappa_{2}$ at region $-L / 2<z<L / 2$ in the borehole can now be expressed as follows:

$$
\kappa_{2}=\frac{\omega}{C_{f}} \sqrt{1-\frac{2}{\kappa R} \frac{H_{1}(\kappa R)}{H_{0}(\kappa R)}}
$$

where $H_{0}$ and $H_{1}$ are Hankel functions of zeroth and first orders, respectively. This relation is derived from the assumption that an oscillatory Poiseuille flow exists in the fracture satisfying the no-slip boundary conditions at $z=\mp L / 2$. We assume the borehole radius $R$ to be $10 \mathrm{~mm}$ and the fluid velocity $C_{f}$ to be $1.5 \mathrm{~km} / \mathrm{s}$. Using equations (6) and (9), we plot the reflection coefficient $A^{-} / A^{+}$and the transmission coefficient $C^{+} / A^{+}$as a function of frequency for different fracture apertures (see Figs. 3, 4). The amplitude of the reflection coefficient increases with fracture aperture, but decreases with frequency. The trends of the transmission coefficient are obviously opposite. 


\section{Shock-Tube Experiment}

\subsection{Experiment with Closed Fracture}

We performed several experiments with an elastic Polyvinyl chloride sample $\left(\mathrm{C}_{\mathrm{p}}=\right.$ $2.3 \mathrm{~km} / \mathrm{s}, \mathrm{C}_{\mathrm{s}}=1.1 \mathrm{~km} / \mathrm{s}$ ) for calibration. The length of the sample is $400 \mathrm{~mm}$. The diameter of the borehole, the probe, and the sample are $10,6.3$ and $76.5 \mathrm{~mm}$, respectively. A typical recording of the trigger channel P2 is given in Fig. 5. Scaling is with respect to the incident pressure. The system is triggered at time $t=0$ by the arrival of the pressure step wave in water. At time $0.535 \mathrm{~ms}$, the reflected wave from the sample top arrives at the transducer P2. Therefore, the wave propagation time from the transducer P2 to the top of the sample is $0.268 \mathrm{~ms}$. At time $0.899 \mathrm{~ms}$, the wave reflected from the free water surface arrives at P2. Also in the borehole, experiments were performed. The two test positions of the mobile pressure probe are 225 and $245 \mathrm{~mm}$ from the sample top, respectively. From the $225 \mathrm{~mm}$ TVD (true vertical depth) measurement, at $0.367 \mathrm{~ms}$, the first arrival of the P-wave is recorded,

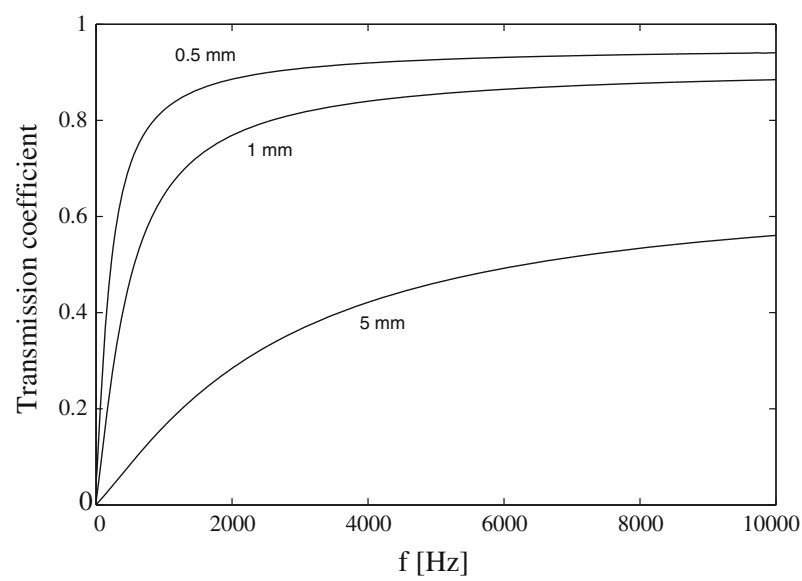

Fig. 4 Transmission coefficient over the fracture at three different fracture apertures

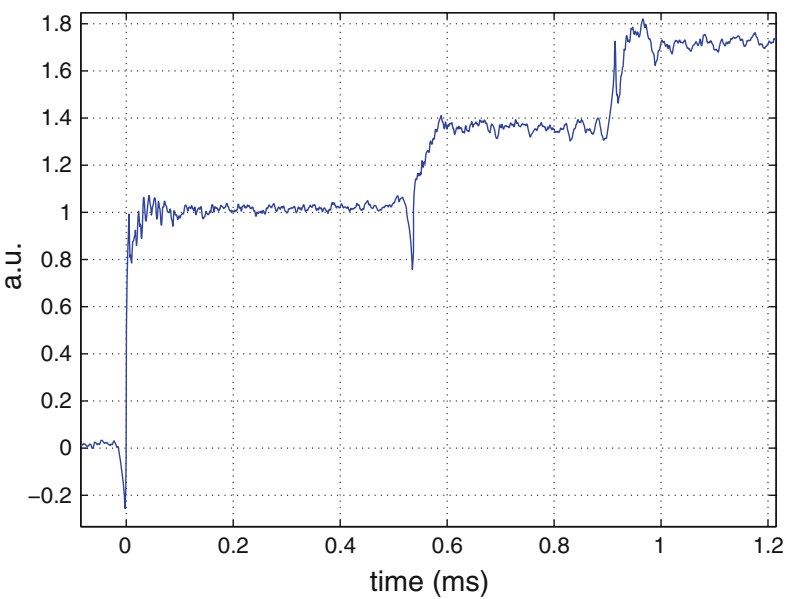

Fig. 5 Recorded pressure signal P2, which is used for triggering the data acquisition system 


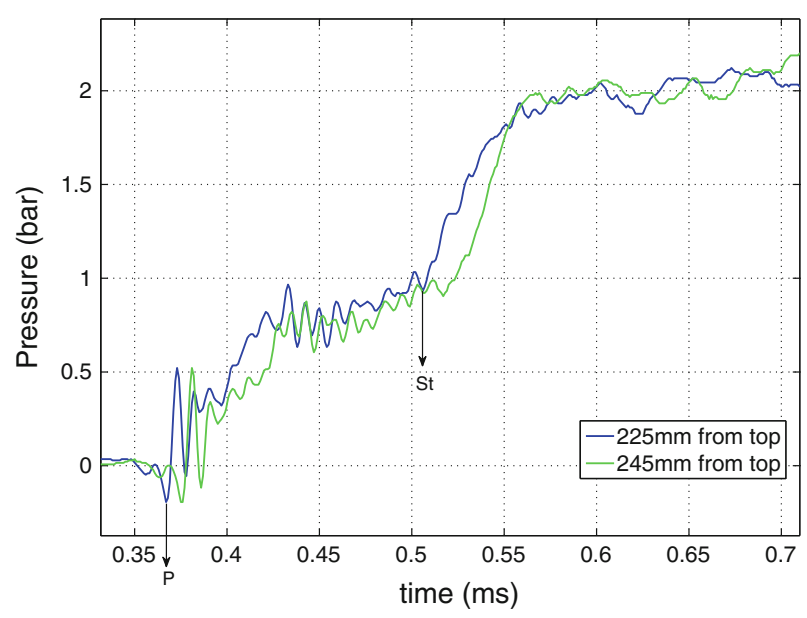

Fig. 6 Recorded pressure signals from the mobile probe P3 at 225- and 245-mm TVD

followed by the arrival of Stoneley wave (St) at $0.506 \mathrm{~ms}$ (see Fig. 6). From the 245-mm TVD recording, we note that it has similar behavior as the 225 TVD measurement, but that the P and St events arrive a little later. Now we can easily deduce the P-wave velocity recorded by the probe inside the borehole to be $2268.5 \mathrm{~m} / \mathrm{s}$, which is in agreement with independent Polyvinyl chloride velocity determination by means of ultrasound. The St velocity is $983.9 \mathrm{~m} / \mathrm{s}$. Obviously, frequency domain analysis would add more insight to the frequency-dependant velocity and attenuation of the individual wave modes (one of which is the St), but that would require sophisticated FFT-Prony-Spectral Ratio analysis, as performed by Chao et al. (2004), to discern the individual wave modes. Here, the focus is on the St mode only.

\subsection{Experiment with Open Fracture}

Now we describe an experiment which has a single horizontal fracture intersecting the sample. The fracture is located at 200-mm TVD. The position of the probe is below the fracture, at 230-mm TVD. The fracture aperture is $5 \mathrm{~mm}$. In reality, 1-2 $\mathrm{mm}$ fractures might be anticipated in the real earth. In that respect, our 5-mm fracture is much larger (taking borehole scaling into consideration). For that reason also a much smaller fracture aperture $(0.1$ $\mathrm{mm}$ ) is studied in our setup (see Fig. 8). We also note that Tang and Cheng (1993) modeled centimeter scale fractures that we want to compare against our experimental results. The recorded pressure profile with a 5-mm fracture is given in Fig. 7. In this figure, the $\mathrm{P}$ wave arrival is at $0.369 \mathrm{~ms}$ and the St arrival is at $0.526 \mathrm{~ms}$. The pressure between 0.423 and 0.526 $\mathrm{ms}$ is dramatically decreasing because of the presence of the fracture. We also note that in the previous Fig. (6) the influence of the (closed) fracture was already visible as a small pressure rise at $t \approx 0.43 \mathrm{~ms}$, indicating that a fracture once created cannot easily be undone. The theoretical predictions have transmission coefficients always below 1, which indicates that there should be a pressure decrease over the fracture. In this experiment, the pressure drop is almost complete, which is much larger than predicted by theory.

We also performed two new experiments with different fracture apertures: 0.1 and $5 \mathrm{~mm}$. The probe is $110 \mathrm{~mm}$ above the fracture which is at 90-mm TVD. In Fig. 8, we notice the arrival of the P-wave at $0.308 \mathrm{~ms}$ and of the St at $0.368 \mathrm{~ms}$. Obviously, the fracture aperture does not affect primary arrivals above the fracture, so that both measurement curves are 


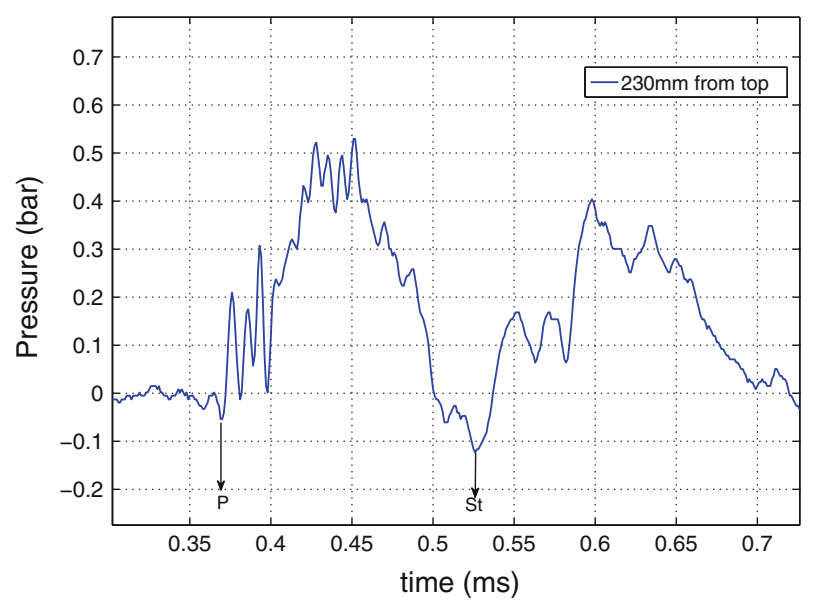

Fig. 7 Recorded pressure signal from the mobile probe P3 at $230 \mathrm{~mm}$ TVD. The fracture aperture is $5 \mathrm{~mm}$. The fracture is at 200-mm TVD

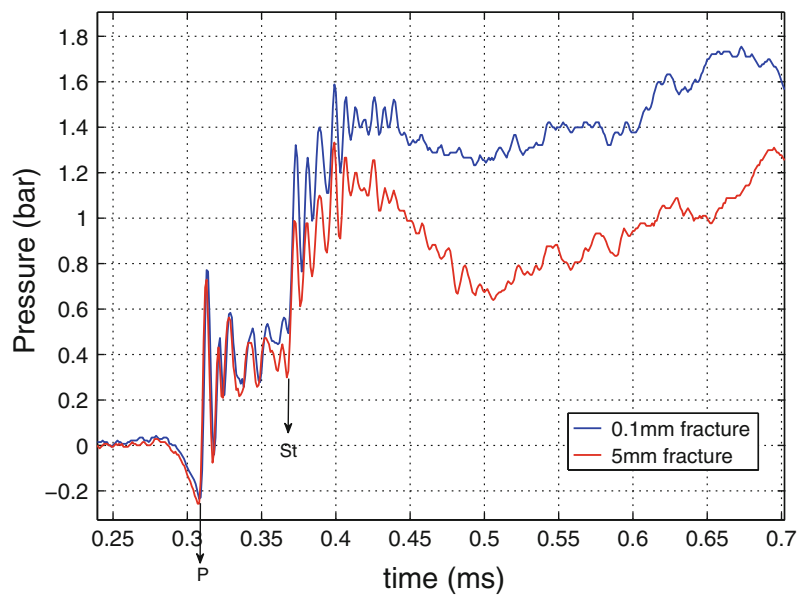

Fig. 8 Recorded pressure signal from the mobile probe P3 at 90-mm TVD. The fracture apertures are 0.1 and $5 \mathrm{~mm}$. The fracture is at 200-mm TVD

indistinguishable. From 0.4 ms onward, both arrivals start to become different. The pressure of the 5-mm fracture experiment decreases significantly after $0.4 \mathrm{~ms}$ compared with the 0.1 $\mathrm{mm}$ fracture aperture experiment. This is probably due to reflection from the fracture that affects the recorded pressure profiles.

\section{Conclusion}

The computation for a single horizontal fracture intersecting a vertical borehole gives a quantitative prediction of reflection and transmission of borehole-guided waves. Three different fracture apertures are used for the calculation. Fracture aperture significantly affects both reflection and transmission coefficients, as was also predicted in the previous literature. 
The shock tube can generate borehole-guided waves in a broad frequency band. Reflection from the water-sample interface and from the free water interface can be recorded by means of a fixed pressure transducer mounted in the wall of the shock tube above the sample in the water layer. We also performed borehole pressure measurements on different fracture apertures at different borehole positions using a specially designed mobile probe. The presence of fractures significantly affects borehole-guided waves. Large fractures increase reflectivity and decrease transmissivity. We found that both pressure above and below the fracture are altered by its presence, thus indicating the potential for fracture detection. Agreement with theory is still only qualitative.

Acknowledgements The first author would like to thank China Scholarship Council for the $\mathrm{PhD}$ fellowship support. The authors are grateful for the technical assistance of Jan Etienne and Karel Heller and to Evert Slob, Gabriel Chao and Karel van Dalen for valuable discussions.

Open Access This article is distributed under the terms of the Creative Commons Attribution License which permits any use, distribution, and reproduction in any medium, provided the original author(s) and the source are credited.

\section{References}

Chao, G., Smeulders, D.M.J., van Dongen, M.E.H.: Shock-induced borehole waves in porous formations: theory and experiments. J. Acoust. Soc. Am. 116(2), 693-702 (2004)

Chilingarian, G.V., Mazzullo, S.J., Rieke, H.H.: Carbonate Reservoir Characterization: A GeologicEngineering Analysis, Part I. pp. 1-35. Elsevier, Amsterdam (1992)

Hornby, B.E., Johnson, D.L., Winkler, K.W., Plumb, R.A.: Fracture evaluation using reflected Stoneley-wave arrivals. Geophysics 54(10), 1274-1288 (1989)

Kostek, S., Johnson, D.L., Randall, C.J.: The interaction of tube waves with borehole fractures, Part I: numerical models. Geophysics 63, 800-808 (1998)

Naimi-Tajdar, R., Han, C., Sepehrnoori, K., Arbogast, T.J., Miller, M.A.: A fully implicit, compositional, parallel simulator for IOR processes in fractured reservoirs. SPE J. 12(3), 367-381 (2007)

Qobi, L., Kuijper, A., Tang, X.M., Strauss, J.: Permeability determination from Stoneley waves in the Ara group carbonates, Oman. GeoArabia 6, 649-666 (2001)

Ramirez, B., Kazemi, H., Al-Kobaisi, M., Ozkan, E., Atan, S.: A critical review for proper use of water/oil/gas transfer functions in dual-porosity naturally fractured reservoirs: Part I. SPE Reserv. Eval. Eng. 12(2), 200-210 (2009)

Rosenbaum, J.H.: Synthetic microseismograms: logging in porous formations. Geophysics 39(1), 14-32 (1974)

Saito, H., Hayashi, K., Iikura, Y.: Detection of formation boundaries and permeable fractures based on frequency-domain Stoneley wave logs. Explor. Geophys. 35(1), 45-50 (2004)

Smeulders, D.M.J., van Dongen, M.E.H.: Wave propagation in porous media containing a dilute gas-liquid mixture: theory and experiments. J. Fluid Mech. 343, 351-373 (1997)

Sniekers, R.W.J.M., Smeulders, D.M.J., van Dongen, M.E.H., Van der Kogel, H.: Pressure wave propagation in a partially water-saturated porous medium. J. Appl. Phys. 66, 4522-4524 (1989)

Tang, X.M., Cheng, C.H.: A dynamic model for fluid flow in open borehole fractures. J. Geophys. Res. 94(B6), 7567-7576 (1989)

Tang, X.M., Cheng, C.H.: Borehole Stoneley wave propagation across permeable structures. Geophys. Prospect. 41(2), 165-187 (1993)

Van der Grinten, J.G., van Dongen, M.E.H., Van der Kogel, H.: A shock tube technique for studying pore pressure propagation in a dry and water-saturated porous medium. J. Appl. Phys. 58, 2937-2942 (1985)

Van der Grinten, J.G., van Dongen, M.E.H., Van der Kogel, H.: Strain and pore pressure propagation in a water-saturated porous medium. J. Appl. Phys. 62, 4682-4687 (1987)

Winkler, K.W., Liu, H.-L., Johnson, D.L.: Permeability and borehole Stoneley waves: comparison between experiment and theory. Geophysics 54(1), 66-75 (1989) 